\title{
The Necessary and Sufficient Conditions of Separability for Multipartite Pure States \\ 1
}

Dafa $\mathrm{Li}^{1, *}$, Xiangrong $\mathrm{Li}^{2}$, Hongtao Huang ${ }^{3}$, Xinxin $\mathrm{Li}^{4}$

${ }^{1}$ Dept of mathematical sciences, Tsinghua University, Beijing 100084 CHINA email:dli@math.tsinghua.edu.cn

${ }^{2}$ Department of Mathematics, University of California, Irvine, CA 92697-3875, USA

${ }^{3}$ Electrical Engineering and Computer Science Department

University of Michigan, Ann Arbor, MI 48109, USA

${ }^{4}$ Dept. of computer science, Wayne State University, Detroit, MI 48202, USA Abstract

In this paper we present the necessary and sufficient conditions of separability for multipartite pure states. These conditions are very simple, and they don't require Schmidt decomposition or tracing out operations. We also give a necessary condition for a local unitary equivalence class for a bipartite system in terms of the determinant of the matrix of amplitudes and explore a variance as a measure of entanglement for multipartite pure states.

Keywords: Entanglement, measure of entanglement, quantum computing, separability.

PACS numbers:03.67.Lx, 03.67.Hk.

\section{Introduction:}

Notation: $M^{+}$is the complex conjugate of transpose of $M$.

Let $|\psi\rangle$ and $|\phi\rangle$ be two pure states of a composite system $A B$ possessed by both Alice and Bob, where system $A(B)$ is called Alice's (Bob's) system. By Nielsen's notation $|\psi\rangle \sim|\phi\rangle$ if and only if $|\psi\rangle$ and $|\phi\rangle$ are locally unitarily equivalent [1]. Let $\rho_{\psi}^{A}$ and $\rho_{\phi}^{A}$ be the states of Alice's system. It is known that $|\psi\rangle \sim|\phi\rangle$ if and only if $\rho_{\psi}^{A}$ and $\rho_{\phi}^{A}$ have the same spectrum of eigenvalues [1] 2. A pure state is separable if and only if it can be written as a tensor product of states of different subsystems. It is also known that a state $|\psi\rangle$ of a bipartite system is separable if and only if it has Schmidt number 1 [3]. Clearly it is essential to do Schmidt decomposition to find the eigenvalues of $\rho_{\psi}^{A}$ and $\rho_{\phi}^{A}$. To obtain a Schmidt decomposition of a pure state $|\psi\rangle$, we need to compute (1) the density operator $\rho_{\psi}^{A B} ;(2)$ the reduced density operator $\rho_{\psi}^{A}$ for system $A ;(3)$ the eigenvalues of $\rho_{\psi}^{A}$. However it is hard to compute roots of a characteristic polynomial of high degree.

Peres presented a necessary and sufficient condition for the occurrence of Schmidt decomposition for a tripartite pure state [4] and showed that the positivity of the partial transpose of a density matrix is a necessary condition for separability [5]. Thapliyal showed that a multipartite pure state is Schmidt decomposable if and only if the density matrices obtained by tracing out any

\footnotetext{
${ }^{1}$ The paper was supported by NSFC(Grant No. 60433050), the fundamental research fund of Tsinghua university NO: JC2003043 and partially by the state key lab. of intelligence technology and system
} 
party are separable [6]. In [7] the local invariants of quantum-bit systems were investigated. In [8] 9] the local symmetry properties and local invariants of pure three-qubit states were discussed, respectively. In [10] the classification of threequbit states was given. Bennett reported measures of multipartite pure-state entanglement in [11. Meyer and Wallach 12 proposed a measure of $n$-qubit pure-state entanglement. Nielsen used the majorization of the eigenvalues of the reduced density operators of a composite system $A B$ to describe the equivalence class under LOCC transformations.

For a multi $(n)$-partite system, in this paper we illustrate the reduced density operators obtained by tracing out the $i$ th subsystem $\rho^{12 \ldots(i-1)(i+1) \ldots n}=$ $\operatorname{tr}_{i}\left(\rho^{12 \ldots n}\right)=M_{i} M_{i}^{+}$, where $i=1,2, \ldots, n$ and $M_{i}$ are the $d^{n-1} \times d$ matrices, of which every entry is an amplitude of the state in question. For a bipartite system $A B$, the reduced density operator $\rho_{\psi}^{A}\left(\rho_{\psi}^{B}\right)=M M^{+}$, where $M$ is the matrix of the amplitudes. Hence $\operatorname{det}\left(\rho_{\psi}^{A}\right)=|\operatorname{det}(M)|^{2}$. However, for a multi $(n)$-partite system, $M_{i}$ are not square. In section 2 , we present a necessary and sufficient condition for separability for a bipartite system in terms of the determinants of all the $2 \times 2$ submatrices of the matrix of the amplitudes. Section 3 contains three versions of the necessary and sufficient separability criterion for a $n$-qubit system. Section 4 is devoted to study the separability of multipartite pure states, and two versions of the necessary and sufficient separability criterion are proposed. Section 5 gives a simple necessary criterion for $|\psi\rangle \sim|\phi\rangle$ for a bipartite system. Section 6 suggests an intuitive measure of multipartite pure-state entanglement.

\section{The separability for a bipartite system}

Let $|\psi\rangle$ be a pure state of a composite system $A B$ possessed by both Alice and Bob. In this section we give a simple and intuitive criterion for the separability. Let $|i\rangle(|j\rangle)$ be the orthonormal basis for system $A(B)$. Then we can write $|\psi\rangle=\sum_{i, j} a_{i j}|i\rangle|j\rangle$, where $\sum_{i j=0}^{n-1}\left|a_{i j}\right|^{2}=1$. Let $M=\left(a_{i j}\right)_{n \times n}$ be the matrix of the amplitudes of $|\psi\rangle$. Then the criterion for the separability is as follows.

$|\psi\rangle$ is separable if and only if the determinants of all the $2 \times 2$ submatrices of $M$ are zero.

This criterion for the separability avoids Schmidt decomposition. To compute the determinants, it needs $n^{2}(n-1)^{2} / 2$ multiplication operations and $n^{2}(n-1)^{2} / 4$ minus operations.

Proof. Suppose that systems $A$ and $B$ have the same dimension $n$. By definition, $|\psi\rangle$ is separable if and only if we can write $|\psi\rangle=\left(\sum_{i=0}^{n-1} x_{i}|i\rangle\right) \otimes$ $\left(\sum_{j=0}^{n-1} y_{j}|j\rangle\right)$, where $\sum_{i=0}^{n-1}\left|x_{i}\right|^{2}=1$ and $=\sum_{j=0}^{n-1}\left|y_{j}\right|^{2}=1$. By tensor product $|\psi\rangle=\sum_{i, j=0}^{n-1} x_{i} y_{j}|i\rangle|j\rangle$. It means that $|\psi\rangle$ is separable if and only if $x_{i} y_{j}=a_{i j}$,

$i, j=0,1, \ldots,(n-1) \ldots \ldots(1)$. Let $m=\left(\begin{array}{cc}a_{i l} & a_{i k} \\ a_{j l} & a_{j k}\end{array}\right)$ be any $2 \times 2$ submatrix of $M$. It is easy to check $\operatorname{det}(m)=a_{i l} a_{j k}-a_{i k} a_{j l}=x_{i} y_{l} x_{j} y_{k}-x_{i} y_{k} x_{j} y_{l}=0$. Therefore if $|\psi\rangle$ is separable then the determinants of all the $2 \times 2$ submatrices 
of $M$ are zero.

Conversely, suppose that the determinants of all the $2 \times 2$ submatrices of $M$ are zero. We can write $M$ in the block form, $M=\left(\begin{array}{c}A_{0} \\ A_{1} \\ \vdots \\ A_{n-1}\end{array}\right)=$ $\left(B_{0}, B_{1}, \ldots, B_{n-1}\right)$, where $A_{i}$ is the $i$ th row and $B_{i}$ is the $i t h$ column of $M$, respectively, $i=0,1, \ldots,(n-1)$. Let $\left|x_{i}\right|^{2}=A_{i} A_{i}^{+} \ldots . .(2)$ and $\left|y_{j}\right|^{2}=B_{j}^{+} B_{j} \ldots . .(3)$, $i, j=0,1, \ldots,(n-1)$, respectively. Under the supposition we can show that the above $x_{i}$ in (2) and $y_{j}$ in (3) satisfy (1). Let us consider the case in which all the $a_{i j}$ are real. It is not hard to extend the result to the case in which all the $a_{i j}$ are complex. We only show $\left|x_{0} y_{0}\right|^{2}=\left|a_{00}\right|^{2}$ and omit the others. From (2) and (3), $\left|x_{0} y_{0}\right|^{2}=A_{0} A_{0}^{+} B_{0}^{+} B_{0}=\left(\sum_{j=0}^{n-1}\left|a_{0 j}\right|^{2}\right)\left(\sum_{i=0}^{n-1}\left|a_{i 0}\right|^{2}\right)=$ $\sum_{i, j=0}^{n-1}\left|a_{0 j}\right|^{2}\left|a_{i 0}\right|^{2}=\sum_{i, j=0}^{n-1}\left|a_{00}\right|^{2}\left|a_{i j}\right|^{2}=\left|a_{00}\right|^{2}$. In the last but one step we use the equality $\left|a_{0 j}\right|^{2}\left|a_{i 0}\right|^{2}=\left|a_{00}\right|^{2}\left|a_{i j}\right|^{2}$, which holds since $\left(\begin{array}{cc}a_{00} & a_{0 j} \\ a_{i 0} & a_{i j}\end{array}\right)$ is a $2 \times 2$ submatrix of $M$. This completes the proof.

Corollary

If $|\psi\rangle$ is separable then $\operatorname{det}(M)=0$.

\section{The separability for a $n$-qubit system}

Let $|\psi\rangle$ be a pure state of a $n$-qubit system. Then we can write $|\psi\rangle=$ $\sum_{i_{1}, i_{2}, \ldots, i_{n} \in\{0,1\}} a_{i_{1} i_{2} \ldots i_{n}}\left|i_{1} i_{2} \ldots i_{n}\right\rangle$. Let the density operator $\rho^{12 \ldots n}=|\psi\rangle\langle\psi|$ and $\rho^{12 \ldots(i-1)(i+1) \ldots n}$ be the reduced density operator obtained by tracing out the $i t h$ qubit. Then $\rho^{12 \ldots(i-1)(i+1) \ldots n}=\operatorname{tr}_{i}\left(\rho^{12 \ldots n}\right)=M_{i} M_{i}^{+}$, where $i=1,2, \ldots, n$ and $M_{i}$ are $2^{(n-1)} \times 2$ matrices of the form $\left(a_{b_{1} b_{2} \ldots b_{i-1} 0 b_{i+1} \ldots b_{n}}, a_{b_{1} b_{2} \ldots b_{i-1} 1 b_{i+1} \ldots b_{n}}\right)$ in which $b_{1}, b_{2}, \ldots, b_{n} \in\{0,1\}$.

For example, let $|\psi\rangle$ be a state of a 3 -qubit system. Then $|\psi\rangle$ can be written as $|\psi\rangle=\sum_{i=0}^{7} a_{i}|i\rangle . M_{3}$ is a $4 \times 2$ matrix $\left(\begin{array}{cc}a_{0} & a_{1} \\ a_{2} & a_{3} \\ a_{4} & a_{5} \\ a_{6} & a_{7}\end{array}\right)$. Each entry of $M_{3}$ is an amplitude of $|\psi\rangle$.

There are three versions of the separability.

Version $1 .|\psi\rangle$ is separable if and only if the determinants of all the $2 \times 2$ submatrices of $M_{1}, M_{2}, \ldots$ and $M_{n}$ are zero.

The proof of version 1 is similar to the one for a bipartite system in section 2 .

Version 2. $|\psi\rangle$ is separable if and only if $a_{i} a_{j}=a_{k} a_{l}$, where $i+j=k+l$ and $i \oplus j=k \oplus l$ where $0 \leq i, j, k, l \leq 2^{n}-1$ are $n$-bit strings and $\oplus$ indicates addition modulo 2 .

For example, 2, 7, 5 and 4 can be written in binary numbers as $010,111,101$ and 100 , respectively. It is well known $010+111($ modulo 2$)=101,101+$ 
$100=001$ (modulo 2 ). Therefore $2+7 \neq 5+4$ (modulo 2 ) though $2+7=5+4=9$.

Using this condition it is easy to verify that states $|W\rangle=1 / \sqrt{n}\left(\left|2^{0}\right\rangle+\left|2^{1}\right\rangle+\right.$ $\ldots+\left|2^{n-1}\right\rangle$ and $|G H Z\rangle=1 / \sqrt{2}\left(\left|0^{(n)}\right\rangle+\left|1^{(n)}\right\rangle\right)$ for a $n$-qubit system [13] are entangled.

Let $i_{1} i_{2} \ldots i_{n}, j_{1} j_{2} \ldots j_{n}, k_{1} k_{2} \ldots k_{n}$ and $l_{1} l_{2} \ldots l_{n}$ be $n$-bit strings of $i, j, k$ and $l$, respectively. Then version 3 is phrased below.

Version 3. $|\psi\rangle$ is separable if and only if $a_{i} a_{j}=a_{k} a_{l}$, where $\left\{i_{t}, j_{t}\right\}=\left\{k_{t}, l_{t}\right\}$, $t=1,2, \ldots, n$.

The following lemma 1 shows that versions 2 and 3 are equivalent to each other.

Lemma 1. $i+j=k+l$ and $i \oplus j=k \oplus l$ if and only if $\left\{i_{t}, j_{t}\right\}=\left\{k_{t}, l_{t}\right\}$, $t=1,2, \ldots, n$.

The proof of lemma 1 is put in appendix A.

We argue version 3 next.

Assume that $|\psi\rangle=\left(x_{0}^{(1)}|0\rangle+x_{1}^{(1)}|1\rangle\right) \otimes\left(x_{0}^{(2)}|0\rangle+x_{1}^{(2)}|1\rangle\right) \otimes \ldots \otimes\left(x_{0}^{(n)}|0\rangle+\right.$ $\left.x_{1}^{(n)}|1\rangle\right)$. By tensor product $x_{i_{1}}^{(1)} x_{i_{2}}^{(2)} \ldots x_{i_{n}}^{(n)}=a_{i_{1} i_{2} \ldots i_{n}}$, where $i_{t}=0,1, t=$ $1,2, \ldots, n$. Then $a_{i} a_{j}=x_{i_{1}}^{(1)} x_{j_{1}}^{(1)} x_{i_{2}}^{(2)} x_{j_{2}}^{(2)} \ldots x_{i_{n}}^{(n)} x_{j_{n}}^{(n)}$ and $a_{k} a_{l}=x_{k_{1}}^{(1)} x_{l_{1}}^{(1)} x_{k_{2}}^{(2)} x_{l_{2}}^{(2)} \ldots x_{k_{n}}^{(n)} x_{l_{n}}^{(n)}$. Explicitly, $a_{i} a_{j}=a_{k} a_{l}$ whenever $\left\{i_{t}, j_{t}\right\}=\left\{k_{t}, l_{t}\right\}, t=1,2, \ldots, n$.

Conversely, suppose that $a_{i} a_{j}=a_{k} a_{l}$ whenever $\left\{i_{t}, j_{t}\right\}=\left\{k_{t}, l_{t}\right\}, t=$ $1,2, \ldots, n$. Let $\left|x_{i_{t}}^{(t)}\right|^{2}=\sum_{i_{1}, \ldots, i_{t-1}, i_{t+1}, ., i_{n} \in\{0,1\}}\left|a_{i_{1} i_{2}, \ldots, i_{n}}\right|^{2}$, where $t=1,2, \ldots, n$. We can show $\left|x_{i_{1}}^{(1)} x_{i_{2}}^{(2)} \ldots . x_{i_{n}}^{(n)}\right|^{2}=\left|a_{i_{1} i_{2} \ldots i_{n}}\right|^{2}$. We only demonstrate the cases of $n=2$ and 3 to give the essential ideas of the general case.

When $n=2$, see section 2 . When $n=3$, see appendix $B$. The two cases suggest that it be simpler to prove $\left|x_{i_{1}}^{(1)} x_{i_{2}}^{(2)} \ldots x_{i_{n}}^{(n)}\right|^{2}=\left|a_{i_{1} i_{2} \ldots i_{n}}\right|^{2}\left(\sum\left|a_{i_{1} i_{2} \ldots i_{n}}\right|^{2}\right)^{n-1}$. Now we finish the argument for the real number case. It is not hard to extend the result to the complex number case.

\section{The separability for a multi $(n)$-partite sys- tem}

Assume that each subsystem has the same dimension $d$. Let $\left|i_{t}\right\rangle$ be the orthonormal basis $|0\rangle,|1\rangle, \ldots,|(d-1)\rangle$ for the $t$ th subsystem. Then any pure state $|\psi\rangle$ can be written as $|\psi\rangle=\sum_{i_{1}, i_{2}, \ldots, i_{n}=0}^{d-1} a_{i_{1} i_{2} \ldots i_{n}}\left|i_{1} i_{2} \ldots i_{n}\right\rangle$. Assume that $|\psi\rangle$ is separable. Then we can write $|\psi\rangle=\left(\sum_{i_{1}=0}^{d-1} x_{i_{1}}^{(1)}\left|i_{1}\right\rangle\right) \otimes\left(\sum_{i_{2}=0}^{d-1} x_{i_{2}}^{(2)}\left|i_{2}\right\rangle\right) \otimes$ $\ldots \otimes\left(\sum_{i_{n}=0}^{d-1} x_{i_{n}}^{(n)}\left|i_{n}\right\rangle\right)$. By tensor product $x_{i_{1}}^{(1)} x_{i_{2}}^{(2)} \ldots . x_{i_{n}}^{(n)}=a_{i_{1} i_{2} \ldots i_{n}}$, where $i_{1}, i_{2}, \ldots, i_{n} \in\{0,1, \ldots,(d-1)\}$.

Let the density operator $\rho^{12 \ldots n}=|\psi\rangle\langle\psi|$ and $\rho^{12 \ldots(i-1)(i+1) \ldots n}$ be the reduced density operator obtained by tracing out the $i t h$ subsystem. Then $\rho^{12 \ldots(i-1)(i+1) \ldots n}=$ $\operatorname{tr}_{i}\left(\rho^{12 \ldots n}\right)=M_{i} M_{i}^{+}$, where $i=1,2, \ldots, n$ and $M_{i}$ are $d^{n-1} \times d$ matrices of the amplitudes of the form

$\left(a_{k_{1} k_{2} \ldots k_{i-1} 0 k_{i+1} \ldots k_{n}}, a_{k_{1} k_{2} \ldots k_{i-1} 1 k_{i+1} \ldots k_{n}}, \ldots, a_{k_{1} k_{2} \ldots k_{i-1}(d-1) k_{i+1} \ldots k_{n}}\right)$, where $k_{1}, k_{2}, \ldots, k_{i-1}, k_{i+1}, \ldots, k_{n} \in$ $\{0,1, \ldots,(d-1)\}$. 
There are two versions of the separability.

Version 1. $|\psi\rangle$ is separable if and only if the determinants of all the $2 \times 2$ submatrices of $M_{1}, M_{2}, \ldots$ and $M_{n}$ are zero.

Version 2. $|\psi\rangle$ is separable if and only if $a_{i_{1} i_{2} \ldots i_{n}} a_{j_{1} j_{2} \ldots j_{n}}=a_{k_{1} k_{2} \ldots k_{n}} a_{l_{1} l_{2} \ldots l_{n}}$, where $\left\{i_{t}, j_{t}\right\}=\left\{k_{t}, l_{t}\right\}, t=1,2, \ldots, n$.

The proof of version 1 is similar to the one for a bipartite system. The proof of version 2 is similar to the one for a $n$-qubit system.

When $n=2$, the criterion is reduced to the one for a bipartite system. When $d=2$, the criterion is reduced to the one for a $n$-qubit system.

\section{A necessary condition for a local unitary equiv- alence class for a bipartite system}

We use the following lemma 2 to establish the necessary condition.

Lemma 2. Let $|\psi\rangle$ be a pure state of a composite system $A B$ possessed by both Alice and Bob. Let $M=\left(a_{j k}\right)_{n \times n}$ be the matrix of the amplitudes of $|\psi\rangle$.

Let $\rho^{A B}=|\psi\rangle\langle\psi|$ and $\rho^{A}=\operatorname{tr}_{B}\left(\rho^{A B}\right)$. Then $|\operatorname{det}(M)|^{2}$ is just the product of the eigenvalues of $\rho^{A}$.

The proof is put in appendix C.

Lemma 2 reveals the relation between the determinant of the matrix of the amplitudes and the eigenvalues of $\rho^{A}$ for a bipartite system.

The corollary of lemma 2

Let $M_{\psi}\left(M_{\phi}\right)$ be the matrix of the amplitudes of a pure state $|\psi\rangle(|\phi\rangle)$ of a composite system $A B$. Then $\left|\operatorname{det}\left(M_{\psi}\right)\right|=\left|\operatorname{det}\left(M_{\phi}\right)\right|$ whenever $|\psi\rangle \sim|\phi\rangle$. That is, $\left|\operatorname{det}\left(M_{\psi}\right)\right|$ is invariant under local unitary operators.

It is well known that it only needs $O\left(n^{3}\right)$ multiplication operations to compute $|\operatorname{det}(M)|$ instead of doing Schmidt decomposition in 1] 2.

For a two-qubit system, let $|\psi\rangle=a|00\rangle+b|01\rangle+c|10\rangle+d|11\rangle$ and $\rho^{12}=|\psi\rangle\langle\psi|$. By lemma $2|a d-b c|^{2}$ is the product of the eigenvalues of $\rho^{1}$. Let $|a d-b c|=\epsilon$.

We can show that $\in$ satisfies $0 \leq \in \leq \frac{1}{2}$ and eigenvalues $\lambda_{ \pm}=\frac{1 \pm \sqrt{1-4 \epsilon^{2}}}{2}$. Hence, $|\psi\rangle \sim \sqrt{\lambda_{+}}|00\rangle+\sqrt{\lambda_{-}}|11\rangle$ or $|\psi\rangle \sim \sqrt{\lambda_{-}}|00\rangle+\sqrt{\lambda_{+}}|11\rangle$.

\section{The variance as a measure of entanglement}

We obtain the necessary and sufficient conditions of separability in sections 2, 3 and 4. Apparently, $\left|a_{i_{1} i_{2} \ldots i_{n}} a_{j_{1} j_{2} \ldots j_{n}}-a_{k_{1} k_{2} \ldots k_{n}} a_{l_{1} l_{2} \ldots l_{n}}\right|$, where $\left\{i_{t}, j_{t}\right\}=$ $\left\{k_{t}, l_{t}\right\}, t=1,2, \ldots, n$., is just a deviation from a product state. It is intuitive to suggest the variance: $\sum\left|a_{i_{1} i_{2} \ldots i_{n}} a_{j_{1} j_{2} \ldots j_{n}}-a_{k_{1} k_{2} \ldots k_{n}} a_{l_{1} l_{2} \ldots l_{n}}\right|^{2}$, where $\left\{i_{t}, j_{t}\right\}=$ $\left\{k_{t}, l_{t}\right\}, t=1,2, \ldots, n$, as a measure of entanglement of $|\psi\rangle$. Let $D_{E}(|\psi\rangle)$ be the measure of entanglement.

$D_{E}(|\psi\rangle)$ has the following properties.

Property 1. $D_{E}(|\psi\rangle)=0$ if and only if $|\psi\rangle$ is separable.

The properties for a two-qubit system 
For a two-qubit system, let $|\psi\rangle=a|00\rangle+b|01\rangle+c|10\rangle+d|11\rangle$. Then $D_{E}(|\psi\rangle)=|a d-b c|^{2}$.

Property 2. The maximum of $D_{E}(|\psi\rangle)=|a d-b c|^{2} \leq(|a d|+|b c|)^{2} \leq$ $\left(\frac{|a|^{2}+|d|^{2}}{2}+\frac{|b|^{2}+|c|^{2}}{2}\right)^{2}=\frac{1}{4}$.

When $a, b, c$ and $d$ are real, by computing extremum it is derived that the maximally entangled states must be of the forms: $x|00\rangle+y|01\rangle-y|10\rangle+x|11\rangle$ or $x|00\rangle+y|01\rangle+y|10\rangle-x|11\rangle$.

Property 3. $|\psi\rangle \sim\left|\psi^{\prime}\right\rangle$ if and only if $D_{E}(|\psi\rangle)=D_{E}\left(\left|\psi^{\prime}\right\rangle\right)$.

Given $|\psi\rangle=a|00\rangle+b|01\rangle+c|10\rangle+d|11\rangle$ and $\left|\psi^{\prime}\right\rangle=a^{\prime}|00\rangle+b^{\prime}|01\rangle+c^{\prime}|10\rangle+$ $d^{\prime}|11\rangle$. Suppose that $|\psi\rangle \sim\left|\psi^{\prime}\right\rangle$. By the necessary condition in section 5, $D_{E}(|\psi\rangle)=D_{E}\left(\left|\psi^{\prime}\right\rangle\right)$.

Conversely, suppose $D_{E}(|\psi\rangle)=D_{E}\left(\left|\psi^{\prime}\right\rangle\right)$. Let us show $|\psi\rangle \sim\left|\psi^{\prime}\right\rangle$. Using Schmidt decomposition, we can write $|\psi\rangle \sim \sqrt{\lambda_{1}}|00\rangle+\sqrt{\lambda_{2}}|11\rangle$, where $\lambda_{1}+$ $\lambda_{2}=1$. As discussed above $|a d-b c|=\sqrt{\lambda_{1}} \sqrt{\lambda_{2}}$. As well using Schmidt decomposition we can write $\left|\psi^{\prime}\right\rangle \sim \sqrt{\rho_{1}}|00\rangle+\sqrt{\rho_{2}}|11\rangle$, where $\rho_{1}+\rho_{2}=1$, and $\left|a^{\prime} d^{\prime}-b^{\prime} c^{\prime}\right|=\sqrt{\rho_{1}} \sqrt{\rho_{2}}$. Thus $\lambda_{1} \lambda_{2}=\rho_{1} \rho_{2}$. Then $\lambda_{1}\left(1-\lambda_{1}\right)=\rho_{1}\left(1-\rho_{1}\right)$. There are two cases. Case 1. $\lambda_{1}=\rho_{1}$. Then $\lambda_{2}=\rho_{2}$. Case 2. $\lambda_{1}+\rho_{1}+1=0$. In the case $\lambda_{2}=\rho_{1}$ and $\lambda_{1}=\rho_{2}$. It means that $|\psi\rangle$ and $\left|\psi^{\prime}\right\rangle$ have the same Schmidt co-efficient for either of the two cases. By factor 5 in [1] 2], $|\psi\rangle \sim\left|\psi^{\prime}\right\rangle$.

Nielsen in 1 showed $\left|\psi^{\prime}\right\rangle \sim\left|\psi^{\prime \prime}\right\rangle$ by calculating eigenvalue, where $\left|\psi^{\prime}\right\rangle=$ $\sqrt{\alpha_{+}}|00\rangle+\sqrt{\alpha_{-}}|11\rangle$, and $\left|\psi^{\prime \prime}\right\rangle=(|00\rangle+|1\rangle(\cos \gamma|0\rangle+\sin \gamma|1\rangle)) / \sqrt{2}$. By property 3 it only needs to check $\sqrt{\alpha_{+}} \sqrt{\alpha_{-}}=\sin \gamma / 2$.

Conclusion

In this paper we have presented the necessary and sufficient conditions of separability for multipartite pure states. These conditions don't require Schmidt decomposition or tracing out operations. By using the conditions it is easy to check whether or not a multipartite pure state is entangled.

Appendix A. The proof of lemma 1

Let $\alpha_{1} \alpha_{2} \ldots \alpha_{n}, \beta_{1} \beta_{2} \ldots \beta_{n}, \delta_{1} \delta_{2} \ldots \delta_{n}$ and $\gamma_{1} \gamma_{2} \ldots \gamma_{n}$ be the $n-$ bit strings of $\alpha$, $\beta, \delta$ and $\gamma$, respectively.

Lemma 1. $\left\{\alpha_{i}, \beta_{i}\right\}=\left\{\delta_{i}, \gamma_{i}\right\}, i=1,2, \ldots, n$, if and only if $\alpha+\beta=\delta+\gamma$ and $\alpha \oplus \beta=\delta \oplus \gamma$, where $\oplus$ indicates addition modulo 2 .

Proof. Suppose $\left\{\alpha_{i}, \beta_{i}\right\}=\left\{\delta_{i}, \gamma_{i}\right\}, i=1,2, \ldots, n$. Since $\alpha+\beta=\left(\alpha_{1}+\right.$ $\left.\beta_{1}\right) 2^{n-1}+\left(\alpha_{2}+\beta_{2}\right) 2^{n-2}+\ldots+\left(\alpha_{n}+\beta_{n}\right)$ and $\delta+\gamma=\left(\delta_{1}+\gamma_{1}\right) 2^{n-1}+\left(\delta_{2}+\right.$ $\left.\gamma_{2}\right) 2^{n-2}+\ldots+\left(\delta_{n}+\gamma_{n}\right)$, by the supposition it is easy to see $\alpha+\beta=\delta+\gamma$. It is straightforward to obtain $\alpha_{1} \alpha_{2} \ldots \alpha_{n} \oplus \beta_{1} \beta_{2} \ldots \beta_{n}=\delta_{1} \delta_{2} \ldots \delta_{n} \oplus \gamma_{1} \gamma_{2} \ldots \gamma_{n}$.

Conversely, suppose $\alpha+\beta=\delta+\gamma$ and $\alpha \oplus \beta=\delta \oplus \gamma$. First let us consider the case where $n=1$. There are three cases.

Case 1. $\alpha_{1}+\beta_{1}=\delta_{1}+\gamma_{1}=0$. This means $\alpha_{1}=\beta_{1}=\delta_{1}=\gamma_{1}=0$.

Case 2. $\alpha_{1}+\beta_{1}=\delta_{1}+\gamma_{1}=1$. This implies $\left\{\alpha_{1}, \beta_{1}\right\}=\left\{\delta_{1}, \gamma_{1}\right\}=\{1,0\}$.

Case 3. $\alpha_{1}+\beta_{1}=\delta_{1}+\gamma_{1}=2$. This says $\alpha_{1}=\beta_{1}=\delta_{1}=\gamma_{1}=1$.

No matter which of the above three cases happens, it yields $\left\{\alpha_{1}, \beta_{1}\right\}=\left\{\delta_{1}\right.$, $\left.\gamma_{1}\right\}$.

Let us consider the case $n$. Since $\alpha+\beta=\delta+\gamma,\left(\alpha_{1}+\beta_{1}\right) 2^{n-1}+\left(\alpha_{2}+\right.$ $\left.\beta_{2}\right) 2^{n-2}+\ldots+\left(\alpha_{n}+\beta_{n}\right)=\left(\delta_{1}+\gamma_{1}\right) 2^{n-1}+\left(\delta_{2}+\gamma_{2}\right) 2^{n-2}+\ldots+\left(\delta_{n}+\gamma_{n}\right)$. Again 
since $\alpha \oplus \beta=\delta \oplus \gamma$, that is, $\alpha_{1} \alpha_{2} \ldots \alpha_{n} \oplus \beta_{1} \beta_{2} \ldots \beta_{n}=\delta_{1} \delta_{2} \ldots \delta_{n} \oplus \gamma_{1} \gamma_{2} \ldots \gamma_{n}$, we obtain $\alpha_{i} \oplus \beta_{i}=\delta_{i} \oplus \gamma_{i}, i=1,2, \ldots, n$. There are two cases.

Case 1. $\alpha_{n} \oplus \beta_{n}=\delta_{n} \oplus \gamma_{n}=1$. In the case $\left\{\alpha_{n}, \beta_{n}\right\}=\left\{\delta_{n}, \gamma_{n}\right\}=\{0,1\}$. Then $\left(\alpha_{1}+\beta_{1}\right) 2^{n-2}+\left(\alpha_{2}+\beta_{2}\right) 2^{n-3}+\ldots+\left(\alpha_{n-1}+\beta_{n-1}\right)=\left(\delta_{1}+\gamma_{1}\right) 2^{n-2}+$ $\left(\delta_{2}+\gamma_{2}\right) 2^{n-3}+\ldots+\left(\delta_{n-1}+\gamma_{n-1}\right) \quad$ and $\alpha_{i} \oplus \beta_{i}=\delta_{i} \oplus \gamma_{i}, i=1,2, \ldots, n-1$. By induction hypothesis $\left\{\alpha_{i}, \beta_{i}\right\}=\left\{\delta_{i}, \gamma_{i}\right\}, i=1,2, \ldots, n-1$.

Case 2. $\alpha_{n} \oplus \beta_{n}=\delta_{n} \oplus \gamma_{n}=0$. There are two subcases.

Subcase 2.1. $\alpha_{n}=\beta_{n}=\delta_{n}=\gamma_{n}=0$ or $\alpha_{n}=\beta_{n}=\delta_{n}=\gamma_{n}=1$. As discussed in case 1 , we can obtain $\left\{\alpha_{i}, \beta_{i}\right\}=\left\{\delta_{i}, \gamma_{i}\right\}, i=1,2, \ldots, n-1$ by induction hypothesis.

Subcase 2.2. $\alpha_{n}=\beta_{n}=1$ and $\delta_{n}=\gamma_{n}=0$ or $\alpha_{n}=\beta_{n}=0$ and $\delta_{n}=\gamma_{n}=1$. Let us consider the former case. In the case $\left(\alpha_{1}+\beta_{1}\right) 2^{n-2}+\left(\alpha_{2}+\beta_{2}\right) 2^{n-3}+$ $\ldots+\left(\alpha_{n-2}+\beta_{n-2}\right) 2+\left(\alpha_{n-1}+\beta_{n-1}+1\right)=$

$\left(\delta_{1}+\gamma_{1}\right) 2^{n-2}+\left(\delta_{2}+\gamma_{2}\right) 2^{n-3}+\ldots+\left(\delta_{n-2}+\gamma_{n-2}\right) 2+\left(\delta_{n-1}+\gamma_{n-1}\right)$.

Since $\alpha_{n-1} \oplus \beta_{n-1}=\delta_{n-1} \oplus \gamma_{n-1}$, either $\alpha_{n-1} \oplus \beta_{n-1}=\delta_{n-1} \oplus \gamma_{n-1}=0$ or 1 causes that one of $\left(\alpha_{n-1}+\beta_{n-1}+1\right)$ and $\left(\delta_{n-1}+\gamma_{n-1}\right)$ is odd and the other is even. It contradicts $\alpha \oplus \beta=\delta \oplus \gamma$.

Appendix B. The separability for a $n$-qubit system

When $n=3$, let us show $\left|x_{i_{1}}^{(1)} x_{i_{2}}^{(2)} x_{i_{3}}^{(3)}\right|^{2}=\left|a_{i_{1} i_{2} i_{3}}\right|^{2}$ when $a_{i} a_{j}=a_{k} a_{l}$, where $\left\{i_{t}, j_{t}\right\}=\left\{k_{t}, l_{t}\right\}, t=1,2,3$. We only illustrate $\left|x_{0}^{(1)} x_{0}^{(2)} x_{0}^{(3)}\right|^{2}=\left|a_{000}\right|^{2}$. Other cases then follow readily. Experientially, it is simpler to prove $\left|x_{0}^{(1)} x_{0}^{(2)} x_{0}^{(3)}\right|^{2}=$ $\left|a_{000}\right|^{2}\left(\sum_{i, j, k \in\{0,1\}}\left|a_{i j k}\right|^{2}\right)\left(\sum_{i, j, k \in\{0,1\}}\left|a_{i j k}\right|^{2}\right)$, where $\left|x_{0}^{(1)}\right|^{2}=\sum_{i, j \in\{0,1\}}\left|a_{0 i j}\right|^{2}$, $\left|x_{0}^{(2)}\right|^{2}=\sum_{k, l \in\{0,1\}}\left|a_{k 0 l}\right|^{2}$ and $\left|x_{0}^{(3)}\right|^{2}=\sum_{p, q \in\{0,1\}}\left|a_{p q 0}\right|^{2}$.

First we show that $a_{0 i j} a_{k 0 l} a_{p q 0}$ can be rewritten as $a_{000} a_{\alpha_{1} \alpha_{2} \alpha_{3}} a_{\delta_{1} \delta_{2} \delta_{3}}$. There are the following four cases.

Case 1. Consider $a_{0 i j} a_{k 0 l}$ and the pairs $\{0, k\},\{i, 0\}$ and $\{j, l\}$. If $j * l=0$, then $a_{0 i j} a_{k 0 l}=a_{000} a_{k i(j+l)}$ since $\{j, l\}=\{0, j+l\}$.

Case 2. Consider $a_{0 i j} a_{p q 0}$ and the pairs $\{0, p\},\{i, q\}$ and $\{j, 0\}$. If $i * q=0$, then $a_{0 i j} a_{p q 0}=a_{000} a_{p(i+q) j}$ since $\{i, q\}=\{0, i+q\}$.

Case 3. Consider $a_{k 0 l} a_{p q 0}$ and the pairs $\{k, p\},\{0, q\}$ and $\{l, 0\}$. If $k * p=0$, then $a_{k 0 l} a_{p q 0}=a_{000} a_{(k+p) q l}$ since $\{k, p\}=\{0, k+p\}$.

Case 4. Otherwise $i=j=l=k=p=q=1$. It is not hard to derive $a_{3} a_{5} a_{6}=a_{1} a_{7} a_{6}=a_{0} a_{7}^{2}$.

Second, let us show that $a_{000} a_{\alpha_{1} \alpha_{2} \alpha_{3}} a_{\delta_{1} \delta_{2} \delta_{3}}$ can be rewritten as $a_{0 i j} a_{k 0 l} a_{p q 0}$. If $a_{000} a_{\alpha_{1} \alpha_{2} \alpha_{3}} a_{\delta_{1} \delta_{2} \delta_{3}}$ is of the forms: $a_{000} a_{0 i j} a_{k 0 l}, a_{000} a_{0 i j} a_{p q 0}$ or $a_{000} a_{k 0 l} a_{p q 0}$, then these forms are desired. Otherwise $a_{000} a_{\alpha_{1} \alpha_{2} \alpha_{3}} a_{\delta_{1} \delta_{2} \delta_{3}}$ must be $a_{0} a_{6} a_{6}$, $a_{0} a_{3} a_{3}, a_{0} a_{5} a_{5}$ or of the form $a_{0} a_{7} a_{r s t}$, which can be rewritten as $a_{2} a_{4} a_{6}, a_{1} a_{2} a_{3}$, $a_{1} a_{4} a_{5}, a_{1} a_{6} a_{r s t}$, respectively. $a_{2} a_{4} a_{6}, a_{1} a_{2} a_{3}$ and $a_{1} a_{4} a_{5}$ are just desired and $a_{1} a_{6} a_{r s t}$ is furthermore rewritten as follows. There are three cases.

Case 1 . In the case $r=0$ or $s=0$, this is desired.

Case 2. In the case $r=s=t=1, a_{1} a_{6} a_{7}=a_{3} a_{5} a_{6}$, desired.

Case 3. In the case $r=s=1$ and $t=0, a_{1} a_{6} a_{6}=a_{2} a_{5} a_{6}$, desired.

Appendix C. The proof of lemma 2

Proof. Suppose that systems $A$ and $B$ have the same dimensions $n$. Let $|\psi\rangle=$ 
$\sum_{i, j=0}^{n-1} a_{i j}|i\rangle|j\rangle$. Then $M=\left(a_{i j}\right)_{n \times n}$. Let density operator $\rho^{A B}=|\psi\rangle\langle\psi|$. Then $\rho^{A B}=\left(\sum_{i, j=0}^{n-1} a_{i j}|i\rangle|j\rangle\right)\left(\sum_{l, k=0}^{n-1} a_{l k}^{*}\left\langle l|\langle k|)=\sum_{i, j=0}^{n-1} \sum_{l, k=0}^{n-1} a_{i j} a_{l k}^{*} \mid i\right\rangle|j\rangle\langle l|\langle k|\right.$

$=\sum_{i, l=0}^{n-1} \sum_{j, k=0}^{n-1} a_{i j} a_{l k}^{*}|i\rangle|j\rangle\langle l|\langle k|$. The reduced density operator for system $A$ is defined by $\rho^{A}=\operatorname{tr}_{B}\left(\rho^{A B}\right)$. Let us compute $\rho^{A}$.

$\rho^{A}=\sum_{i, l=0}^{n-1} \sum_{j, k=0}^{n-1} a_{i j} a_{l k}^{*}|i\rangle\langle l| \delta_{k j}$ (where $\delta_{k j}=1$ when $k=j$. Otherwise 0.) $=\sum_{i, l=0}^{n-1} \sum_{j=0}^{n-1} a_{i j} a_{l j}^{*}|i\rangle\left\langle l\left|=\sum_{i, l=0}^{n-1}\left(\sum_{j=0}^{n-1} a_{i j} a_{l j}^{*}\right)\right| i\right\rangle\langle l|$. Let $A_{i}=$ $\left(a_{i 0}, a_{i 1}, \ldots . a_{i(n-1)}\right)$, that is, the $i$ th row of $A$. Then $\sum_{j=0}^{n-1} a_{i j} a_{l j}^{*}=A_{i} A_{l}^{+}$. Finally $\rho^{A}=\sum_{i, l=0}^{n-1} A_{i} A_{l}^{+}|i\rangle\langle l|=\left(\begin{array}{c}A_{0} \\ A_{1} \\ \vdots \\ A_{n-1}\end{array}\right)\left(A_{0}^{+}, A_{1}^{+}, \ldots, A_{n-1}^{+}\right)=M M^{+}$. Thus $\operatorname{det}\left(\rho^{A}\right)=|\operatorname{det}(M)|^{2}$. Hence $|\operatorname{det}(M)|^{2}$ is just the product of the eigenvalues of $\rho^{A}$. Q.E.D.

\section{References}

[1] M.A. Nielsen, Phys. Rev. Lett. 83, 436(1999).

[2] A. Peres, Quantum theory: Concepts and methods (Kluwer Academic Dordrecht, 1993). P. 123.

[3] M. A. Nielsen and I. L. Chuang, Quantum computation and quantum information (Cambridge University Press, Cambridge, England, 2000). p. 109.

[4] A. Peres, Phys. Lett. A 202, 16 (1995).

[5] A. Peres, Phys. Rev. Lett. 77, 1413 (1996).

[6] A. V. Thapliyal, Phys. Rev. A 59, 3336 (1999).

[7] M. Grassl et al., Phys. Rew. A. 58 (1998) 1833-1839.

[8] H. A. Carteret and A. Sudbery, J. Phys. A: Math. Gen. 33 (2000)4981-5002.

[9] A. Sudbery, J. Phys. A: Math. Gen. 34 (2001)643-652.

[10] A. Acin et al., Phys. Rew. Lett. 85 (2000) 1560-1563.

[11] C. H. Bennett et al., Phys. Rev. A., 63(2000) 012307.

[12] D. A. Meyer and N. R. Wallach, J. of mathematical physics 43 (2002) 4273-4278.

[13] W. Dür et al., Phys. Rev. A., 62(2000)062314. 